\title{
Molecular Cores in Taurus: Evolution and Dynamics
}

\author{
Lei Qian ${ }^{1}$ Di Li $^{1}$ and Paul Goldsmith ${ }^{2}$ \\ ${ }^{1}$ National Astronomical Observatories, Chinese Academy of Sciences, \\ Beijing 100012, P. R. China \\ email: lqian@nao.cas.cn \\ ${ }^{2}$ Jet Propulsion Laboratory, California Institute of Technology, CA, USA
}

\begin{abstract}
The evolution and dynamics of the molecular cores traced by ${ }^{13} \mathrm{CO} J=1 \rightarrow 0$ emission in the Taurus molecular cloud are studied. We performed a systematic examination of the distribution and dynamic state of cores in a large contiguous region, as well as the core ages, which lie between $10^{6}$ and $10^{7}$ years. The core velocity dispersion (CVD), which is the variance of the core velocity difference $\delta v$, exhibits a power-law behavior as a function of the apparent separation $L$, i.e. CVD $(\mathrm{km} / \mathrm{s}) \propto L(\mathrm{pc})^{0.7}$, with similarities to Larson's law for the velocity dispersion of the gas.
\end{abstract}

Keywords. ISM: clouds - ISM: molecules — ISM: individual (Taurus) - turbulence

\section{Core Ages and Core Velocity Dispersion (CVD)}

A sample of of 765 cores (588 cores relevant for this work) are found in the ${ }^{13} \mathrm{CO}$ $(J=1 \rightarrow 0)$ data cube of the Taurus molecular cloud (Goldsmith et al. 2008), where a core is defined as a Gaussian component in the ${ }^{13} \mathrm{CO}$ data cube (x-y-v cube). Cores with peak intensity greater than $5 \sigma$ are picked out, where $\sigma$ is the background noise level in the data cube.

During the core evolution, $\mathrm{HI}$ converts to $\mathrm{H}_{2}$, the core ages can then be estimated by using the fractional $\mathrm{HI}$ abundance. The core ages are found to be in between $10^{6}$ and $10^{7}$ years.

There are $588 \times(588-1) / 2$ unique core pairs. We calculate the dispersion of the velocity difference $\delta v$ of these pairs and the core velocity dispersion CVD $\equiv<\delta v^{2}>^{1 / 2}$ versus the apparent separation between cores $L$. The relation between the CVD and $L$ can be fitted with a power law as CVD $(\mathrm{km} / \mathrm{s})=0.2 L(\mathrm{pc})^{0.7}+0.2$ for $L$ between 0 and $10 \mathrm{pc}$, similar to Larson's law. The mean value of the CVD for separation $L>10 \mathrm{pc}$ is $1.18 \mathrm{~km} / \mathrm{s}$.

\section{Discussion}

The low velocity dispersion among cores and the close similarity between CVD vs. $L$ relation and Larson's law suggest that molecular cores condense out of the diffuse gas and move with the flow field, without additional energy from star formation or significant impact from converging flows.

Acknowledgment. This work is partly supported by China Ministry of Science and Technology under State Key Development Program for Basic Research (2012CB821800).

\section{Reference}

Goldsmith, P. F., Heyer, M., Narayanan, G., Snell, R., Li, D., \& Brunt, C. 2008, ApJ, 680, 428 